\title{
Comparison of Efficacy of Pembrolizumab between Epstein-Barr Virus-Positive and -Negative Relapsed or Refractory Non-Hodgkin Lymphomas
}

\author{
Seok-Jin Kim, MD, PhD 1 \\ Jiyeon Hyeon, MD, PhD² \\ Inju Cho, $\mathrm{MD}^{2}$ \\ Young Hyeh Ko, MD, PhD² \\ Won Seog Kim, MD, PhD ${ }^{1}$
}

\begin{abstract}
${ }^{1}$ Division of Hematology-Oncology, Department of Medicine, ${ }^{2}$ Department of Pathology, Samsung Medical Center, Sungkyunkwan University School of Medicine, Seoul, Korea
\end{abstract}

Correspondence: Won Seog Kim, MD, PhD Division of Hematology/Oncology, Department of Medicine, Samsung Medical Center, Sungkyunkwan University School of Medicine, 81 Irwon-ro, Gangnam-gu, Seoul 06351, Korea

Tel: 82-2-3410-6548

Fax: 82-2-3410-1754

E-mail: wskimsmc@skku.edu

Received April 2, 2018

Accepted July 17, 2018

Published Online July 20, 2018

*Seok-Jin Kim and Jiyeon Hyeon contributed equally to this work.

\begin{abstract}
Purpose
Pembrolizumab, a programmed cell death protein 1 (PD1) inhibitor inhibits the interplay between PD1 of T-cell and programmed cell death ligand 1 (PDL1) on tumor cells. Although pembrolizumab has been tried to various subtypes of non-Hodgkin lymphoma (NHL), realworld data about the efficacy of pembrolizumab in NHL patients are limited.
\end{abstract}

\section{Materials and Methods}

We analyzed the outcome of 30 relapsed or refractory NHL patients treated with pembrolizumab, and compared the outcome between Epstein-Barr virus (EBV)-positive and negative subtypes because EBV infection of tumor cells can upregulate PDL1 expression.

\section{Results}

Seven patients with EBV-positive NHL showed a response including NK/T-cell lymphoma $(6 / 14,44 \%)$ and primary mediastinal B-cell lymphoma (1/4, 25\%) whereas EBV-negative subtypes did not respond such as diffuse large B-cell lymphoma and T-lymphoblastic lymphoma. We also evaluated PDL1 expression using tumor tissue of 76 patients. High PDL1 expression (positive staining of $>50 \%$ of tumor cells) was more frequent in NK/T-cell lymphoma and primary mediastinal B-cell lymphoma than other subtypes. Thus, PDL1 expression was significantly higher in EBV-positive $(18 / 32,56 \%)$ than EBV-negative NHL $(4 / 38$, $11 \%, p<0.001$ ). Furthermore, NK/T-cell lymphoma patients with high PDL1 expression showed a higher response $(4 / 6,67 \%)$ than those with low PDL1 expression $(1 / 5,20 \%)$.

\section{Conclusion}

Pembrolizumab could be useful as a salvage treatment for relapsed or refractory EBV-positive NHL, especially NK/T-cell lymphoma. However, its efficacy in EBV-negative NHL with low or absent PDL1 expression is still not clear although pembrolizumab could be a potential treatment option for relapsed or refractory NHL.

\section{Introduction}

Non-Hodgkin lymphoma (NHL) represents a heterogeneous group of malignancies with distinct clinical and pathological features. New treatments have improved the outcomes of NHL, but a substantial number of patients still relapse and require multiple treatments because only a limited number of patients respond to salvage treatment and the response is usually of short duration [1,2]. Furthermore, as
Key words

Lymphoma, Pembrolizumab, PDL1, Epstein-Barr virus 
to PD1 on the surface of effector T-cells transduces inhibitory signals to the T cells, allowing tumor cells to escape antitumor immunity [4]. Therefore, inhibition of this interplay between PD1 and PDL1 can reverse this immune escape and enhance T-cell cytotoxicity against tumor cells. Currently, two monoclonal IgG4 anti-PD1 antibodies, nivolumab and pembrolizumab, are approved by the Food and Drug Administration (FDA) as salvage treatment for solid cancers such as melanoma and non-small cell lung cancer. The FDA has also granted accelerated approval to nivolumab and pembrolizumab for the treatment of relapsed or refractory classical Hodgkin lymphoma (HL). However, data concerning the use of pembrolizumab in patients with NHL are limited because its efficacy in relapsed or refractory NHL has not been determined. In our institute, we have used pembrolizumab to treat patients with various subtypes of relapsed or refractory NHL, and already reported the exciting outcome of one patient with relapsed NK/T-cell lymphoma (NKTCL) in a previous multicenter retrospective study [5]. Since January 2016, we have used pembrolizumab as a salvage treatment for thirty patients with relapsed or refractory NHL. In this study, we retrospectively analyzed their treatment outcome to illustrate our real-world experience with pembrolizumab treatment in relapsed or refractory NHL outside of clinical trials.

\section{Materials and Methods}

\section{Patients and treatment}

Thirty consecutive patients with relapsed or refractory NHL were treated with pembrolizumab at the Samsung Medical Center between January 2016 and September 2017. Informed consent for the use of pembrolizumab was obtained from all patients. Because the cost of pembrolizumab is not reimbursed by health insurance in Korea, a fixed low dose of $100 \mathrm{mg}$ was administered to 27 patients while three patients received the recommended dose of $200 \mathrm{mg}$. The treatment cycle was repeated every 3 weeks. The response was assessed using 2-deoxy-2-fluorine-18-fluoro-D-glucose positron emission tomography integrated with computed tomography and computed tomography according to standard criteria. In most patients, the first response evaluation was performed after the second or third cycle of treatment [6]. If signs of rapid deterioration were observed, response evaluation was performed earlier than the planned date. For patients with NKTCL, blood Epstein-Barr virus (EBV) DNA titer was also quantified as previously reported as an indirect biomarker of the extent of disease [7].

\section{Assessment of PDL1 expression in NHL}

We assessed PDL1 expression of tumor cells by immunohistochemistry (IHC) to evaluate the extent of PDL1 expression in various subtypes of NHL. Thus, PDL1 expression was assessed in relapsed or refractory NHL patients $(n=76)$ for whom paraffin-embedded tissue blocks were available for IHC. Most tissue samples were from archived tissue samples in our lymphoma registry that were obtained as routine clinical practice at the time of diagnosis $(n=71)$, and only five cases were from obtained at relapse or progression. After IHC, we analyzed the proportion of cells in each tumor subtype that expressed PDL1 to determine whether PDL1 expression differed according to subtype of NHL. We also evaluated the EBV positivity of tumor cells, correlating the results of EBV in situ hybridization with PDL1 expression for each subtype of NHL. These 76 patients included 21 patients treated with pembrolizumab who were analyzed for response evaluation. Thus, we could evaluate the PDL1 expression of tumor cells in patients receiving pembrolizu-mab to determine whether the response to pembrolizumab was correlated with PDL1 expression.

\section{IHC for PDL1 and EBV positivity}

Two assays including monoclonal antibodies against PDL1 were used: the Ventana PDL1 (SP142) assay (1:25, rabbit antihuman PDL1/CD274 monoclonal antibody, clone SP142, Ventana, Tucson, AZ) and the PDL1 IHC 22C3 pharmDx assay, which includes the murine 22C3 anti-human PDL1 antibody (Dako, Carpinteria, CA). PDL1 IHC using the Ventana PDL1 assay was performed on an automated immunostainer (Benchmark, Ventana) according to the manufacturer's instructions. In some cases, PDL1 IHC was performed using the PDL1 IHC 22C3 pharmDx kit using a Link 48 autostainer according to the manufacturer's instructions. Because it was difficult to identify tumor cells precisely when observing only hematoxylin and eosin (H\&E) stained slides, PDL1 expression on tumor cells was investigated by comparing H\&E slides. The proportion of PDL1-positive cells was estimated as a percentage of total tumor cells; tumor cells typically showed membranous staining with a variable component of cytoplasmic staining. The percentage of tumor cells positively stained for PDL1 was determined by pathologists. Because there are no established criteria for high PDL1 expression in lymphoma, we used the cutoff value of $50 \%$ which was suggested by the manufacturer's instructions of PDL1 IHC 22C3 pharmDx kit [8]. Thus, PDL1 expression was defined as follows: No expression (PDL1-positive tumor cells $<1 \%$ ), low expression (PDL1-positive tumor cells 1\%-49\%), and high expression (PDL1-positive tumor cells $\geq 50 \%$ ). 
Table 1. Clinical characteristics of patients at the time of pembrolizumab treatment

\begin{tabular}{|c|c|c|c|c|c|}
\hline & $\begin{array}{c}\text { Total } \\
(n=30)\end{array}$ & $\begin{array}{c}\text { DLBCL } \\
(\mathbf{n}=\mathbf{1 0})\end{array}$ & $\begin{array}{c}\text { PMBCL } \\
(n=4)\end{array}$ & $\begin{array}{c}\text { NKTCL } \\
(\mathrm{n}=14)\end{array}$ & $\begin{array}{c}\text { T-LBL } \\
(n=2)\end{array}$ \\
\hline \multicolumn{6}{|l|}{ Age (yr) } \\
\hline$\leq 60$ & 22 & 7 & 4 & 9 & 2 \\
\hline$>60$ & 8 & 3 & 0 & 5 & 0 \\
\hline \multicolumn{6}{|l|}{ Sex } \\
\hline Male & 21 & 7 & 1 & 11 & 2 \\
\hline Female & 9 & 3 & 3 & 3 & 0 \\
\hline \multicolumn{6}{|l|}{ Performance status } \\
\hline ECOG $0 / 1$ & 9 & 1 & 3 & 4 & 1 \\
\hline $\mathrm{ECOG} \geq 2$ & 21 & 9 & 1 & 10 & 1 \\
\hline \multicolumn{6}{|l|}{ Stage } \\
\hline $\mathrm{I} / \mathrm{II}$ & $2 / 2$ & $0 / 0$ & $1 / 1$ & 2 & 0 \\
\hline III/ IV & $0 / 26$ & $0 / 10$ & $0 / 2$ & 12 & 2 \\
\hline \multicolumn{6}{|l|}{ No. of extranodal involvements } \\
\hline $0 / 1$ & 5 & 1 & 2 & 2 & 0 \\
\hline$\geq 2$ & 25 & 9 & 2 & 12 & 2 \\
\hline \multicolumn{6}{|l|}{ Serum LDH } \\
\hline Normal & 10 & 2 & 1 & 5 & 0 \\
\hline Increased & 20 & 8 & 3 & 9 & 2 \\
\hline \multicolumn{6}{|l|}{ IPI } \\
\hline Low/Low-intermediate & $4 / 2$ & $0 / 1$ & $2 / 0$ & $2 / 0$ & $0 / 1$ \\
\hline High-intermediate/High & $8 / 16$ & $1 / 8$ & $1 / 1$ & $5 / 7$ & $1 / 0$ \\
\hline No. of previous treatments, median (range) & $4(1-10)$ & $5(2-10)$ & $4(3-6)$ & $3(1-7)$ & $2(2-3)$ \\
\hline Previous SCT & 14 & 3 & 2 & 6 & 2 \\
\hline Autologous & 9 & 3 & 2 & 4 & 0 \\
\hline Allogeneic & 4 & 0 & 0 & 2 & 1 \\
\hline Both & 1 & 0 & 0 & 0 & 1 \\
\hline Time to pembrolizumab, median (mo) & 17.0 & 12.2 & 33.7 & 19.0 & 19.9 \\
\hline \multicolumn{6}{|l|}{ EBV } \\
\hline Positive & 15 & 0 & 1 & 14 & 0 \\
\hline Negative & 15 & 10 & 3 & 0 & 2 \\
\hline
\end{tabular}

DLBCL, diffuse large B-cell lymphoma; PMBCL, primary mediastinal B-cell lymphoma; NKTCL, NK/T-cell lymphoma; T-LBL, T-lymphoblastic lymphoma; ECOG, Eastern Cooperative Oncology Group; LDH, lactate dehydrogenase; IPI, International Prognostic Index; SCT, stem cell transplantation; EBV, Epstein-Barr virus.

\section{Study parameters}

The response of each patient was classified based on the best response during pembrolizumab treatment, regardless of the final outcome. The overall response rate (ORR) was based on the number of patients who achieved a complete response $(\mathrm{CR})$ or a partial response $(\mathrm{PR})$. The toxicity profiles of pembrolizumab were determined based on review of the medical records. The number of previous treatments was defined as the total number of cycles of treatment from the first-line chemotherapy to the last chemotherapy before pembrolizumab. Radiotherapy or autologous or allogeneic stem cell transplantation (SCT) were not included in the number of previous treatments because they were used as adjuvant or consolidation therapy. The characteristics of patients including age, performance status, stage, and absolute lymphocyte count were recorded at the time of pembrolizumab treatment, not at the time of diagnosis. Time to pembrolizumab was defined as the interval between the date of diagnosis and the date of the first infusion of pembrolizumab. Post-pembrolizumab survival was defined as the time between the date of first infusion of pembrolizumab and the date of last follow-up or death from any cause. The participants' disease status and survival were updated on December 31, 2017. 


\section{Ethical statement}

The study was approved by the Institutional Review Board of Samsung Medical Center (IRB No. 2017-11-105) and performed in accordance with the principles of the Declaration of Helsinki. The informed consent was waived.

\section{Results}

\section{Characteristics of patients before pembrolizumab}

The median age of patients at the time of pembrolizumab treatment was 49 years (range, 18 to 80 years) and most were men $(n=27)$. The histologic subtypes of tumor were diffuse large B-cell lymphoma (DLBCL; $\mathrm{n}=10$ ), primary mediastinal large B-cell lymphoma (PMBCL; $n=4)$, NKTCL $(n=14)$, and T-cell lymphoblastic lymphoma (T-LBL; $\mathrm{n}=2$ ). Most patients had stage IV disease $(n=26)$ with widespread organ involvement ( $\geq 2$ sites of extranodal involvement, $n=25$ ) (Table 1 ). The majority of patients had been heavily treated before pembrolizumab treatment: the median number of previous chemotherapy treatments excluding SCT was 4 (range, 1 to 10 ), and 16 patients had undergone autologous or allogeneic SCT (Table 1). Five patients had relapsed after allogeneic SCT, including one patient who underwent haploidentical SCT. All patients with DLBCL and T-LBL showed negativity for EBV whereas all cases of NKTCL and one case of PMBCL were positive for EBV (Table 1).

\section{Response to pembrolizumab}

All patients with DLBCL rapidly progressed during pembrolizumab treatment and died. Therefore, more than half the patients discontinued pembrolizumab after their first cycle (median number of cycle, 1 ; range, 1 to 4 ). The two patients with T-LBL had relapsed after haploidentical and unrelated allogeneic SCT and received pembrolizumab as a salvage treatment. However, their disease rapidly progressed after the first of second cycle of pembrolizumab. Of the four patients with PMBCL, case 1 showed a PR, and completed the 12th cycles of pembrolizumab (Fig. 1). She is currently in the state of ongoing treatment. On the contrary to other tumor subtypes, six NKTCL patients responded (5 CR, 1 PR; ORR, 44\%). Case 1 who had relapsed after unrelated allogeneic $\mathrm{SCT}$, showed a CR: this case was included in a previous study [5]. Since then, he has continued to receive pembrolizumab up to 16 cycles, and has shown no clinical or radiologic evidence of disease relapse during treatment (Fig. 1). Case 2, an 80-year-old man who was diagnosed with stage IV NKTCL only received a half-dose of SMILE (steroid, methotrexate, ifosfamide, L-asparaginase, and etoposide) chemotherapy because he was elderly and unsuitable for intensified chemotherapy. After his third cycle of SMILE chemotherapy, his disease progressed with an elevation in blood EBV DNA titer (90,568 copies/mL). However, he achieved CR with an undetectable level of blood EBV DNA after the third cycle of pembrolizumab and completed 12 cycles. Three patients (case 3-5) also continued to receive pembrolizumab up to 19th cycle (Fig. 1). Case 6 showed a PR after the second cycle of pembrolizumab, but the patient refused further treatment for financial reasons; his disease progressed, and he died (Fig. 1). Most patients who showed disease progression after one to three cycles of pembrolizumab died.

\section{Adverse events during pembrolizumab treatment}

Case 1 with NKTCL developed a grade II skin rash after the third cycle of pembrolizumab treatment. The biopsy of skin lesion showed hydropic degeneration and basal vacuolization consistent with graft-versus-host disease (GVHD). Although the time to onset of skin lesion was longer than 1 year because he underwent allogeneic SCT from a matched unrelated donor 10.6 months before pembrolizumab treatment, we reported this event as an acute GVHD in a previous study [5]. The reason was that he did not have any sign of acute GVHD before pembrolizumab and his skin manifestation was not consistent with chronic GVHD. His skin lesions improved with corticosteroid treatment and further therapy was unhindered. In contrast, cases 1 and 2 with T-LBL did not develop any sign of GVHD despite having undergone haploidentical and unrelated allogeneic SCT 11.0 and 6.2 months before pembrolizumab treatment. Case 9 with NKTCL involving the small intestine developed bowel perforation after the first cycle of pembrolizumab and died as a result of sepsis (Fig. 1).

\section{Association of PDL1 with EBV positivity}

PDL1 expression was evaluated in archived tumor tissue samples from 76 patients with NHL including 21 patients treated with pembrolizumab. The proportion of tumors with high PDL1 expression differed according to the subtype of NHL. Thus, high PDL1 expression was more frequent in NKTCL $(15 / 28,54 \%)$, PMBCL $(4 / 6,67 \%)$, and peripheral T-cell lymphoma (PTCL; $2 / 7,29 \%)$ than in DLBCL $(2 / 18$, $11 \%)$ and T-LBL $(0 / 8,0 \%)$. Although the numbers were small, high PDL1 expression was observed in subcutaneous panniculitis-like T-cell lymphoma and angioimmunoblastic T-cell lymphoma unlike primary central nervous system (CNS) DLBCL (0/3), mantle cell lymphoma (0/2), and ente- 
$\operatorname{NKTCL}(\mathrm{n}=14)$

\begin{tabular}{|c|c|c|c|c|c|c|c|c|c|c|c|c|c|c|c|c|c|c|c|c|c|c|c|}
\hline No. & Sex/age & EBV & PDL1 & Dose & 1 & 2 & 3 & 4 & \begin{tabular}{l|l}
5 & 6
\end{tabular} & 7 & \begin{tabular}{l|l}
8 & 9
\end{tabular} & \begin{tabular}{l|l|}
9 & 10
\end{tabular} & 11 & 12 & 13 & 14 & 15 & 16 & 17 & 18 & 19 & $\begin{array}{l}\text { Best } \\
\text { response }\end{array}$ & $\begin{array}{l}\text { Survival } \\
\text { status }\end{array}$ \\
\hline 1 & $\mathrm{M} / 51$ & + & H & 100 & & & & & & & & & & & & & & & & & & CR & Alive \\
\hline 2 & $\mathrm{M} / 80$ & + & H & 100 & & & & & & & & & & & & & & & & & & CR & Alive \\
\hline 3 & $\mathrm{M} / 53$ & + & NA & 100 & & & & & & & & & & & & & & & & & & CR & Alive \\
\hline 4 & $F / 47$ & + & L & 100 & & & & & & & & & & & & & & & & & & $C R$ & Alive \\
\hline 5 & $\mathrm{M} / 47$ & + & H & 100 & & & & & & & & & & & & & & & & & & CR & Alive \\
\hline 6 & $\mathrm{M} / 71$ & + & H & 200 & & & & & & & & & & & & & & & & & & $P R$ & Dead \\
\hline 7 & $\mathrm{M} / 60$ & + & NA & 100 & & & & & & & & & & & & & & & & & & PD & Dead \\
\hline 8 & $\mathrm{~F} / 56$ & + & L & 100 & & & & & & & & & & & & & & & & & & PD & Dead \\
\hline 9 & M/61 & + & H & 100 & & & & & & & & & & & & & & & & & & PD & Dead \\
\hline 10 & $F / 51$ & + & L & 100 & & & & & & & & & & & & & & & & & & PD & Dead \\
\hline 11 & $M / 32$ & + & L & 100 & & & & & & & & & & & & & & & & & & PD & Dead \\
\hline 12 & $\mathrm{M} / 53$ & + & H & 100 & & & & & & & & & & & & & & & & & & PD & Dead \\
\hline 13 & $\mathrm{M} / 32$ & + & L & 100 & & & & & & & & & & & & & & & & & & PD & Dead \\
\hline 14 & M/60 & + & NA & 100 & & & & & & & & & & & & & & & & & & PD & Dead \\
\hline
\end{tabular}

$\operatorname{PMBCL}(\mathrm{n}=4)$

\begin{tabular}{|c|c|c|c|c|c|c|c|c|c|c|c|c|c|c|c|c|c|c|c|c|c|c|c|c|c|}
\hline No. & Sex/age & EBV & PDL1 & Dose & 1 & 2 & 3 & 4 & 5 & 6 & 7 & 8 & 9 & 10 & 11 & 12 & 13 & 14 & 15 & 16 & 17 & 18 & 19 & $\begin{array}{c}\text { Best } \\
\text { response }\end{array}$ & $\begin{array}{c}\text { Survival } \\
\text { status }\end{array}$ \\
\hline 1 & $F / 33$ & + & H & 100 & & & & & & & & & & & & & & & & & & & & PR & Alive \\
\hline 2 & $\mathrm{M} / 20$ & - & $\mathrm{H}$ & 200 & & & & & & & & & & & & & & & & & & & & PD & Alive \\
\hline 3 & $F / 31$ & - & NA & 100 & & & & & & & & & & & & & & & & & & & & $P D$ & $N A$ \\
\hline 4 & $\mathrm{~F} / 18$ & - & NA & 100 & & & & & & & & & & & & & & & & & & & & PD & Dead \\
\hline
\end{tabular}

\section{DLBCL $(n=10)$}

\begin{tabular}{|c|c|c|c|c|c|c|c|c|c|c|c|c|c|c|c|c|c|c|c|c|c|c|c|c|c|}
\hline №. & Sex/age & EBV & PDL1 & Dose & 1 & 2 & 3 & 4 & 5 & 6 & 7 & 8 & 9 & 10 & 11 & 12 & 13 & 14 & 15 & 16 & 17 & 18 & 19 & $\begin{array}{c}\text { Best } \\
\text { response }\end{array}$ & $\begin{array}{c}\text { Survival } \\
\text { status }\end{array}$ \\
\hline 1 & $F / 43$ & - & No & 100 & & & & & & & & & & & & & & & & & & & & PR & Dead \\
\hline 2 & $\mathrm{M} / 49$ & - & No & 100 & & & & & & & & & & & & & & & & & & & & $\overline{P D}$ & Dead \\
\hline 3 & $\mathrm{M} / 44$ & - & No & 100 & & & & & & & & & & & & & & & & & & & & PD & Dead \\
\hline$\overline{4}$ & $\mathrm{M} / 43$ & - & $\mathrm{H}$ & 100 & & & & & & & & & & & & & & & & & & & & PD & Dead \\
\hline 5 & M/67 & - & NA & 100 & & & & & & & & & & & & & & & & & & & & PD & Dead \\
\hline 6 & $\mathrm{M} / 66$ & - & NA & 100 & & & & & & & & & & & & & & & & & & & & PR & Dead \\
\hline 7 & $\mathrm{M} / 56$ & - & NA & 100 & & & & & & & & & & & & & & & & & & & & PD & Dead \\
\hline 8 & $F / 48$ & - & L & 100 & & & & & & & & & & & & & & & & & & & & PD & Dead \\
\hline 9 & $F / 32$ & - & L & 100 & & & & & & & & & & & & & & & & & & & & PD & Dead \\
\hline 10 & $\mathrm{M} / 72$ & - & H & 100 & & & & & & & & & & & & & & & & & & & & $\begin{array}{l}\mathrm{PD} \\
\end{array}$ & Dead \\
\hline
\end{tabular}

$\mathrm{T}-\mathrm{LBL}(\mathrm{n}=2)$

\begin{tabular}{|c|c|c|c|c|c|c|c|c|c|c|c|c|c|c|c|c|c|c|c|c|c|c|c|c|c|}
\hline No. & Sex/age & EBV & PDL1 & Dose & 1 & 2 & 3 & 4 & 5 & 6 & 7 & 8 & 9 & 10 & 11 & 12 & 13 & 14 & 15 & 16 & 17 & 18 & 19 & $\begin{array}{c}\text { Best } \\
\text { response }\end{array}$ & $\begin{array}{l}\text { Survival } \\
\text { status }\end{array}$ \\
\hline 1 & $\mathrm{M} / 45$ & - & No & 200 & & & & & & & & & & & & & & & & & & & & PD & Alive \\
\hline 2 & $\mathrm{M} / 26$ & - & No & 100 & & & & & & & & & & & & & & & & & & & & PD & Dead \\
\hline
\end{tabular}

Fig. 1. Clinical outcome and treatment cycles of NK/T-cell lymphoma (NKTCL), primary mediastinal B-cell lymphoma (PMBCL), diffuse large B-cell lymphoma (DLBCL), and T-lymphoblastic lymphoma (T-LBL). EBV, Epstein-Barr virus; PDL1, programmed cell death ligand 1; H, high; CR, complete response; NA, not available; L, low; PR, partial response; PD, progressive disease. 


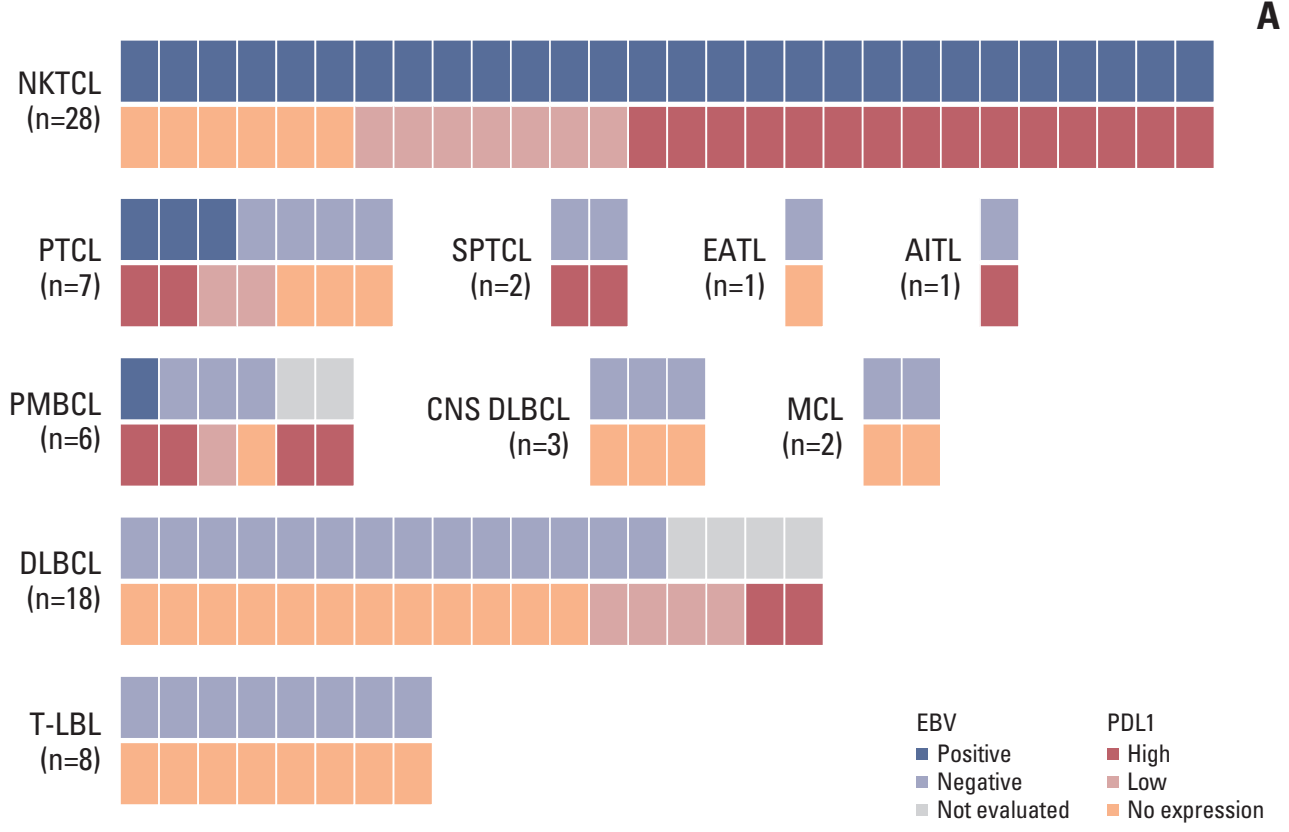

A

B

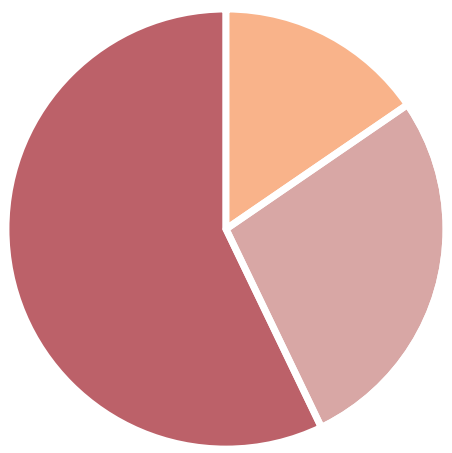

EBV-positive NHL

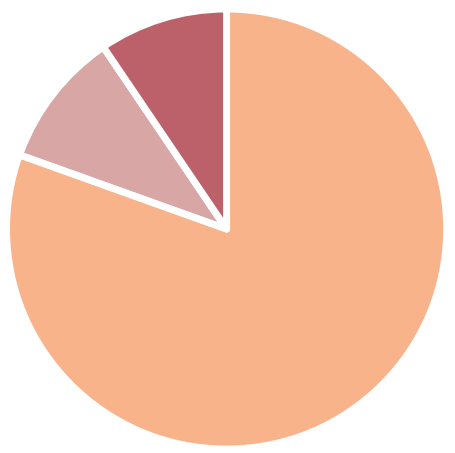

EBV-negative NHL

Fig. 2. (A) Comparison of programmed cell death ligand 1 (PDL1) expression in 76 patients with non-Hodgkin lymphoma shows different frequencies of high ( $\geq 50 \%$ of PDL1-positive tumor cells), low (1\%-49\% of PDL1-positive tumor cells), and no expression. High PDL1 expression is more frequent in NK/T-cell lymphoma (NKTCL; 15/28, 54\%) and primary mediastinal B-cell lymphoma (PMBCL; 4/6, 67\%) than in peripheral T-cell lymphoma (PTCL; 2/7, 28\%), diffuse large B-cell lymphoma (DLBCL; 2/18, 11\%), and T-lymphoblastic lymphoma (T-LBL; 0/8, 0\%). No case of PDL1 high expression is observed in primary central nervous system diffuse large B-cell lymphoma (CNS DLBCL; 0/3), mantle cell lymphoma (MCL, 0/2), or enteropathy-associated T-cell lymphoma (EATL; 0/1). However, subcutaneous panniculitis-like T-cell lymphoma (SPTCL; 2/2) and angioimmunoblastic T-cell lymphoma (AITL; 1/1) show high PDL1 expression. (B) Epstein-Barr virus (EBV)-positive non-Hodgkin lymphoma (NHL) shows a significantly higher proportion of high PDL1 expression (18/32, 56\%) than EBV-negative NHL $(4 / 38,11 \%, \mathrm{p}<0.001)$. 
Table 2. EBV positivity and response to pembrolizumab treatment

\begin{tabular}{|c|c|c|c|}
\hline & \multirow{2}{*}{$\begin{array}{c}\text { Total } \\
(\mathrm{n}=30)\end{array}$} & \multicolumn{2}{|c|}{ EBV } \\
\hline & & Positive (n=15) & Negative $(n=15)$ \\
\hline \multicolumn{4}{|l|}{ Subtype } \\
\hline DLBCL & 10 & 0 & 10 \\
\hline PMBCL & 4 & 1 & 3 \\
\hline NKTCL & 14 & 14 & 0 \\
\hline T-LBL & 2 & 0 & 2 \\
\hline \multicolumn{4}{|l|}{ PDL1 expression } \\
\hline High & 10 & 7 & 3 \\
\hline Low & 7 & 5 & 2 \\
\hline No expression & 5 & 0 & 5 \\
\hline Not evaluated & 8 & 3 & 5 \\
\hline \multicolumn{4}{|l|}{ Response } \\
\hline Complete response & 5 & 5 & 0 \\
\hline Partial response & 2 & 2 & 0 \\
\hline Stable disease & 0 & 0 & 0 \\
\hline Progressive disease & 23 & 8 & 15 \\
\hline
\end{tabular}

EBV, Epstein-Barr virus; DLBCL, diffuse large B-cell lymphoma; PMBCL, primary mediastinal B-cell lymphoma; NKTCL, NK/T-cell lymphoma; T-LBL, T-lymphoblastic lymphoma; PDL1, programmed cell death ligand 1.

ropathy-associated T-cell lymphoma (0/1) (Fig. 2A). Although six patients were not evaluated for EBV in situ hybridization including DLBCL $(\mathrm{n}=4)$ and PMBCL $(\mathrm{n}=2)$, EBVpositivity was more frequent in NKTCL $(28 / 28,100 \%)$ and PTCL $(3 / 7,43 \%)$ than other subtypes. EBV-positive NHL included a significantly higher proportion of tumors with high PDL1 expression (18/32, 56\%) compared with EBV-negative NHL $(4 / 38,11 \%$, p < 0.001) (Fig. 2B). Thus, these results suggest the association of EBV-positivity with PDL1 expression although EBV-positivity was not evaluated in four cases of high PDL1 expression due to lack of available tissue (DLBCL and PMBCL) (Fig. 2A).

\section{Association of PDL1 and EBV positivity with treatment outcome}

When we dichotomized 30 patients receiving pembrolizumab into two groups according to the EBV positivity, seven patients with EBV-positive tumor responded to pembrolizumab (7/15) whereas all patients with EBV-negative tumor failed to respond $(0 / 15)$ (Table 2). Thus, NKTCL $(6 / 14,44 \%)$ and PMBCL $(1 / 4,25 \%)$ showed higher ORR than DLBCL $(0 / 10,0 \%)$ and T-LBL $(0 / 2,0 \%)$. This higher response to pembrolizumab was associated with more frequent expression of PDL1 in EBV-positive tumors. Indeed, aforementioned case 1 of PMBCL who had EBV-positive disease and high PDL1 expression showed a PR to pembrolizumab (Fig. 1). The different proportions of high PDL1 expression in different subtypes of NHL tumors correlated with their response to pembrolizumab. Of 21 pembrolizumab-treated patients whose PDL1 expression was analyzed, five cases with high PDL1 expression responded to pembrolizumab $(5 / 10,50 \%)$ whereas only two cases with low PDL1 expression responded $(2 / 11,18 \%)$; however, this difference was not significant $(\mathrm{p}=0.183$ ). Even in patients with NKTCL, the ORR was different between those with high $(4 / 6,67 \%)$ and low PDL1 expression $(1 / 5,20 \%)$. For example, case 6 of NKTCL with high PDL1 expression showed a PR with undetectable levels of EBV DNA in blood after pembrolizumab treatment (Fig. 3A), whereas case 10 with low PDL1 expression did not respond (Fig. 3B). However, this difference of response based on PDL1 expression could not be statistically analyzed due to small number of patients.

\section{Discussion}

In our study, pembrolizumab was used in different NHL subtypes, and their response to pembrolizumab was different. Thus, the ORR of NKTCL $(6 / 14,44 \%)$ was higher than that of PMBCL $(1 / 4,25 \%)$, DLBCL $(0 / 10,0 \%)$, or T-LBL $(0 / 2$, $0 \%$ ). Although the number of patients in each subtype was small, our findings are consistent with those of a previous case series reporting outstanding outcomes of pembrolizu- 

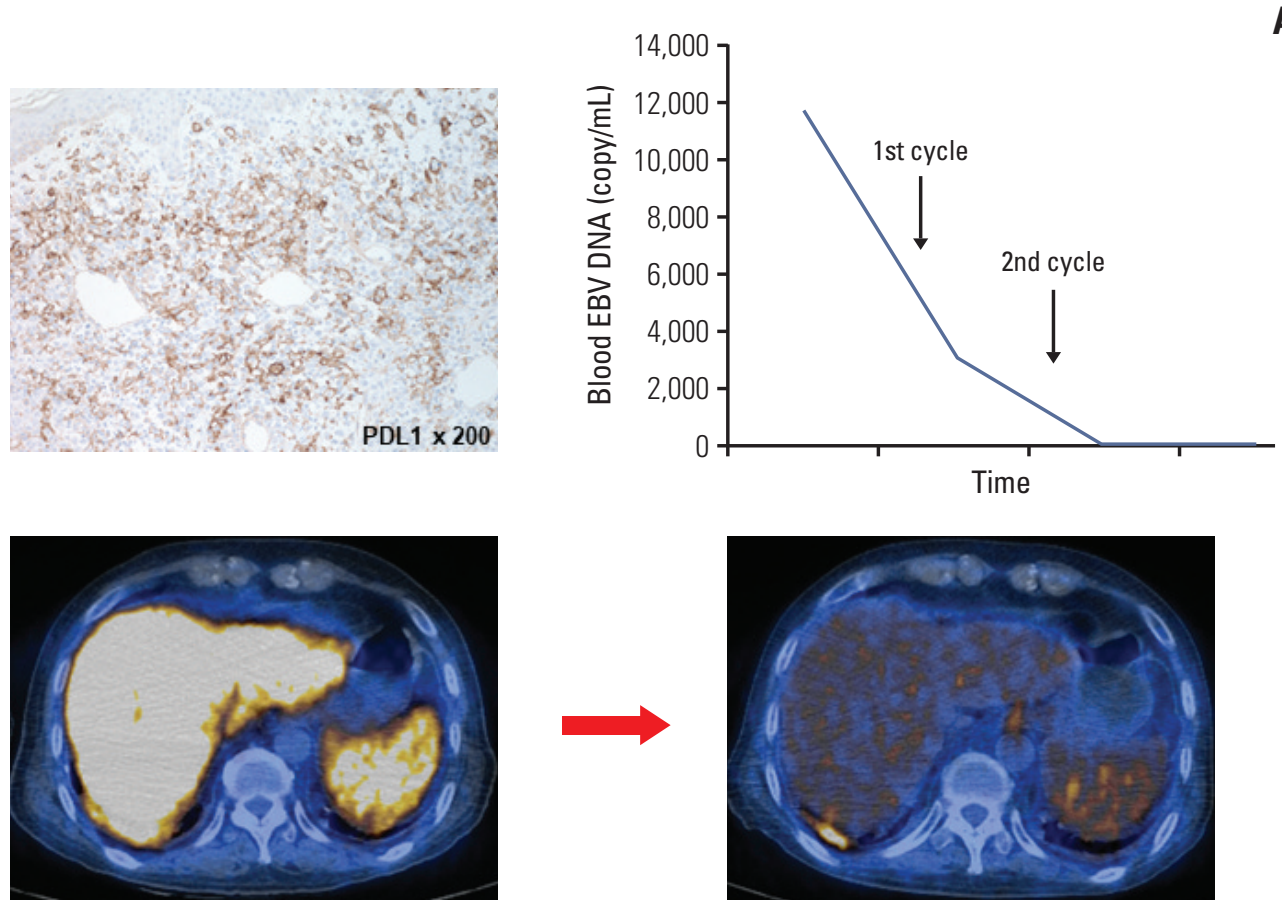

A
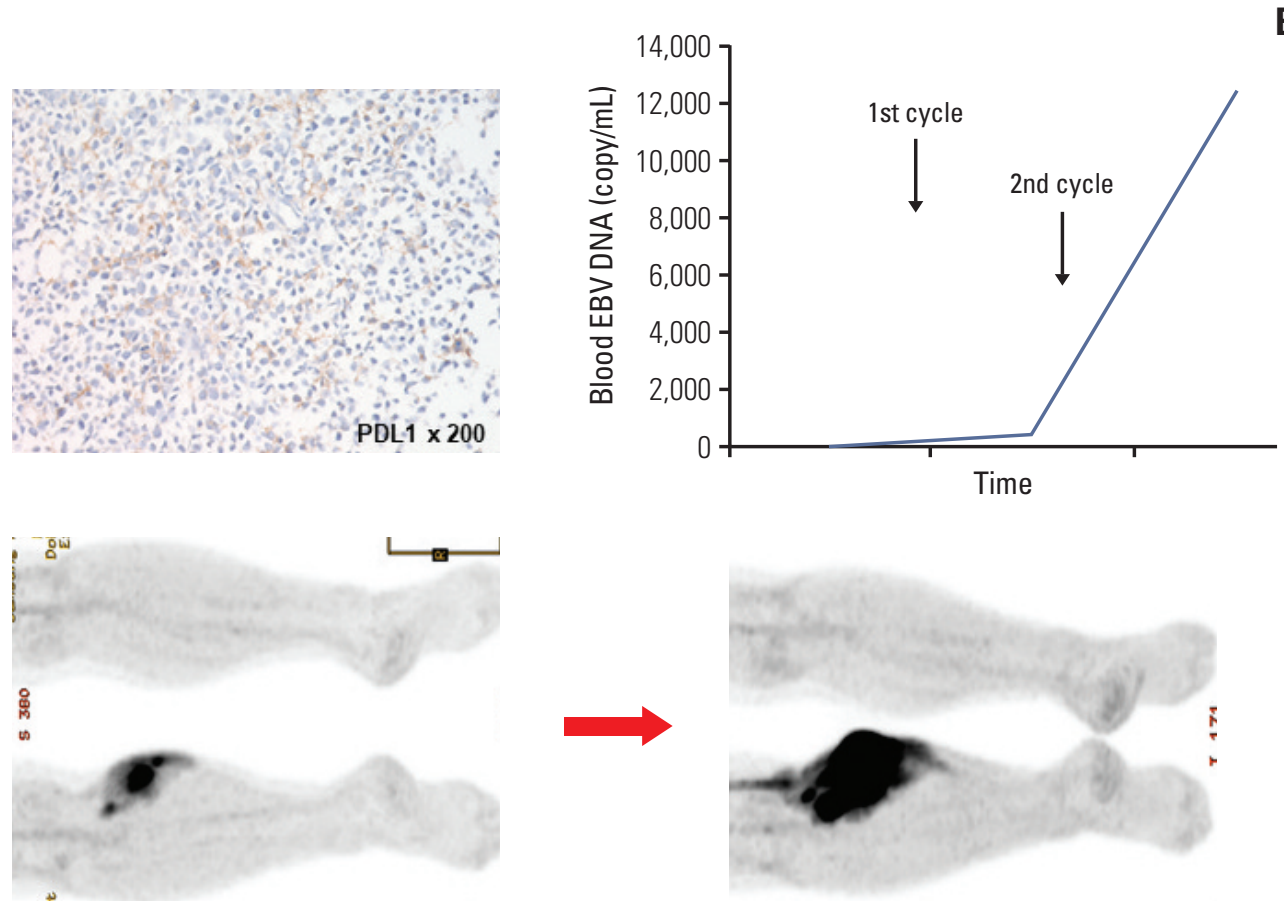

Fig. 3. (A) Immunohistochemistry for programmed cell death ligand 1 (PDL1) shows $\geq 50 \%$ of tumor cells positively stained (brown membranous staining) in a case of extranodal NK/T-cell lymphoma (top left); decrease in blood Epstein-Barr virus (EBV) DNA level after pembrolizumab treatment (top right); decrease in fluorodeoxyglucose (FDG) uptake in liver and spleen after pembrolizumab treatment (bottom). (B) Immunohistochemistry for PDL1 shows $<1 \%$ of tumor cells positively stained in a case of extranodal NK/T-cell lymphoma (top left); increase in blood EBV DNA level even after pembrolizumab treatment (top right); increase in the extent and intensity of FDG uptake in a soft tissue mass in an extremity after pembrolizumab treatment (bottom). 
Table 3. Pembrolizumab in relapsed or refractory non-Hodgkin lymphoma

\begin{tabular}{|c|c|c|c|c|c|c|}
\hline Study & Author & Disease & No. & Dose & Response & ORR \\
\hline Case series & Kwong et al. [5] & NKTCL & 7 & 100 mg every $3 \mathrm{wk}$ & $5 \mathrm{CR}, 2 \mathrm{PR}$ & $100 \%$ \\
\hline Case report & Lai et al. [9] & NKTCL & 1 & $2 \mathrm{mg} / \mathrm{kg}$ every $3 \mathrm{wk}$ & $\begin{array}{l}\text { CR: alive } \\
\text { without relapse }\end{array}$ & - \\
\hline Case report & Chan et al. [10] & ALCL & 1 & $2 \mathrm{mg} / \mathrm{kg}$ every $3 \mathrm{wk}$ & $\mathrm{CR}$ & - \\
\hline Case report & Chan et al. [13] & $\begin{array}{l}\text { Double-hit } \\
\text { lymphoma }\end{array}$ & 1 & $\begin{array}{l}\text { Pembrolizumab } 100 \text { mg } \\
\text { every } 3 \text { wk plus } \\
\text { lenalidomide } 15 \mathrm{mg} \text { daily }\end{array}$ & CR & - \\
\hline Phase $1 b$ trial & $\begin{array}{l}\text { Zinzani } \\
\text { et al. [11] }\end{array}$ & PMBCL & 18 & $200 \mathrm{mg}$ every $3 \mathrm{wk}$ & $2 \mathrm{CR}, 5 \mathrm{PR}$ & $\begin{array}{l}41 \% \text { (7 of } 17 \\
\text { evaluable patients) }\end{array}$ \\
\hline Phase II & Ding et al. [23] & $\begin{array}{l}\text { CLL (16) or } \\
\text { Richter } \\
\text { transformation (9) }\end{array}$ & 25 & 200 mg every 3 wk & $\begin{array}{l}\text { CLL: no response } \\
\text { Transformed } \\
\text { CLL: } 2 \text { CR/2 PR }\end{array}$ & $\begin{array}{l}\text { CLL: } 0 \%(0 / 16) \\
\text { Transformed } \\
\text { CLL: } 44 \%(4 / 9)\end{array}$ \\
\hline Phase II & $\begin{array}{r}\text { Nastoupil } \\
\text { et al. [12] }\end{array}$ & $\begin{array}{l}\text { Follicular } \\
\text { lymphoma }\end{array}$ & 30 & $\begin{array}{l}200 \mathrm{mg} \text { every } 3 \mathrm{wk} \\
\text { plus rituximab } 375 \mathrm{mg} / \mathrm{m}^{2} \\
\mathrm{D} 1,8,15,22\end{array}$ & CR $60 \%$ & $80 \%$ \\
\hline
\end{tabular}

ORR, overall response rate; NKTCL, NK/T-cell lymphoma; CR, complete response; PR, partial response; ALCL, anaplastic large cell lymphoma; PMBCL, primary mediastinal B-cell lymphoma; CLL, chronic lymphocytic leukemia.

mab treatment in NKTCL patients (Table 3). Actually, pembrolizumab as an off-label treatment for relapsed or refractory T- or NK-cell NHL achieved CR $[5,9,10]$. Of the patients with PMBCL, case 1 who was EBV positive with high PDL1 expression responded to pembrolizumab (Fig. 1). However, the other three patients with PMBCL, including one patient with high PDL1 expression, failed to respond. In a previous phase $1 \mathrm{~b}$ study, the ORR to pembrolizumab was $41 \%(7 / 17)$ in relapsed or refractory PMBCL [11]. Although our response rate in PMBCL was inferior to these phase $1 b$ results, the number of patients in our study was too small to draw definite conclusions about the efficacy of pembrolizumab in PMBCL. On the other hand, a recent phase II study using pembrolizumab plus rituximab reported $80 \%$ of ORR in relapsed or refractory follicular lymphoma [12]. This suggests the role of pembrolizumab as a combination treatment with other drugs. Indeed, a patient with double-hit lymphoma receiving pembrolizumab and lenalidomide achieved CR [13]. In our study, although not all patients responded to pembrolizumab, six cases of NKTCL did respond. Therefore, given that NKTCL usually follows an aggressive clinical course after relapse and there is currently no effective salvage treatment for NKTCL, our findings suggested that pembrolizumab could be a useful treatment option for relapsed or refractory NKTCL.

This different response of different NHL subtypes to pembrolizumab could be related to their different levels of PDL1 expression. Thus, our study analyzed the extent of PDL1 expression in 76 patients with NHL including 21 patients who received pembrolizumab. The frequency of high PDL1 expression was very rare in patients with DLBCL $(2 / 18,11 \%)$ and mantle cell lymphoma $(0 / 2,0 \%)$ in our study. Previous studies also reported the absence of PDL1 expression in mantle cell lymphoma [14,15]. Although those studies showed higher frequency of PDL1 expression in DLBCL than that of our study, it might be related with low cutoff value $(5 \%)$ for PDL1 expression (Table 4). Actually, a study analyzing 1,253 patients with DLBCL showed the same incidence of PDL1 expression $(132 / 1,253,11 \%)$ as ours and the study used $30 \%$ of cutoff value [16]. Thus, the poor response to pembrolizumab in DLBCL patients of our study could be explained the low frequency of PDL1 expression. The main mechanism of PDL1 overexpression was amplification of chromosome 9p24.1, and is commonly observed in patients with HL [17]. However, the rearrangement of 9p24.1 leading to PDL1 overexpression was more frequently found in PMBCL and primary CNS DLBCL $[18,19]$. Our study also showed frequent PDL1 expression in PMBCL comparable to that of previous studies [14,15]. However, three cases of primary CNS DLBCL showed no expression of PDL1 unlike a previous study reporting 38\% in Western patients [20]. This might be related with ethnic difference as well as a different cutoff value $(50 \%$ vs. $1 \%$ ). Thus, it should be evaluated in the future study using a larger study population. Our study showed high PDL1 expression in more than $50 \%$ of patients with NKTCL, and previous studies also reported high frequency of PDL1 expression [21,22]. This could be related with that tumor cells of NKTCL are invariably infected with EBV because EBV 


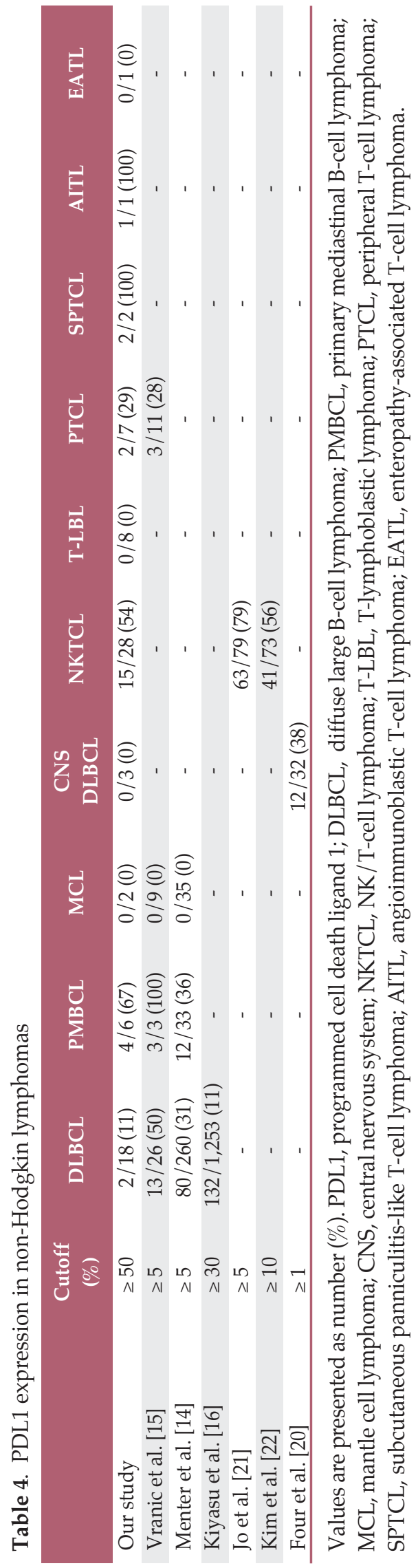

infection of tumor cells and activation of the inflammationrelated JAK/STAT pathway also could upregulate PDL1 expression [24]. Other subtypes of NHL also had EBV-positive tumor cells although its frequency was lower than NKTCL. The frequency of EBV-positivity was known as approximately $10 \%$ in DLBCL, thus, some cases of EBV-positive DLBCL might have PDL1 expression [16]. However, considering poor response to pembrolizumab in DLBCL patients, even in case of high PDL1 expression, pembrolizumab should be very cautiously used in DLBCL patients (Fig. 1). Thus, it should be determined whether PDL1 is expressed on lymphoma cells before pembrolizumab treatment is initiated. Although only two patients with T-LBL received pembrolizumab, it cannot be recommended for this disease entity because PDL1 expression is absent in T-LBL. Overall, these results suggest that PDL1 expression on lymphoma cells might be useful as a biomarker predicting the response to pembrolizumab.

However, our study has several limitations as follows. First, we defined high PDL1 expression based on the cutoff value of $50 \%$ because there are no established criteria for high PDL1 expression in lymphoma. Thus, the frequency of high PDL1 expression in lymphoma patients might become different according to the cutoff values. However, a previous study for the development of a companion diagnostic PDL1 IHC assay for pembrolizumab therapy in non-small-cell lung cancer (NSCLC) showed the treatment effect of pembrolizumab was substantial in patients with $\geq 50 \%$ of PDL1-postitive tumor cells [25]. Furthermore, a phase III trial with pembrolizumab for NSCLC also reported a significant association of longer survival outcome with PDL1 expression on at least $50 \%$ of tumor cells [26]. Therefore, our cutoff value could be used to define high PDL1 expression in lymphoma although further studies with a larger study population should be performed to determine the correlation of response to pembrolizumab with PDL1 expression in lymphoma patients. Second, we used two types of antibodies to assess PDL1 expression. This might influence the results of PDL1 expression in our study. However, our pathology confirmed no difference of efficacy in analyzing the PDL1 expression between two types of assay. Furthermore, given the range of low and high PDL1 expression was wide (1\%-49\% vs. $\geq 50 \%)$, the use of two types of IHC assay might not complicate interpretation of IHC results. Third, most tissues samples for PDL1 expression were obtained from the time of diagnosis. Considering PDL1 expression might be different between diagnosis and relapse/progression, some patients showing the discrepancy of response and PDL1 expression might be associated with this limitation. Fourth, a substantial number of patients discontinued to receive pembrolizumab due to disease progression in our study (Fig. 1). Although it could be interpreted as a lack of efficacy in those patients, the probability of atypical 
response patterns mimicking disease progression could exist because immune-reaction inducing tumor-flare or pseudoprogression could be found in the treatment with pembrolizumab in other solid tumors. Thus, response evaluation and the assessment of disease status should be more careful in lymphoma patients receiving pembrolizumab as the refinement of the Lugano Classification Response Criteria for Lymphoma was proposed in the era of therapy with immune checkpoint inhibitor [27]. Fifth, the number of each subtype was small, especially T-LBL, and there is lack of data about the outcome of other subtypes such as mantle cell lymphoma. Thus, future study with a larger study population including more subtypes should be warranted. Lastly, the dosage of pembrolizumab given to most patients $(100 \mathrm{mg}$ every 3 weeks) was lower than that recommended (200 $\mathrm{mg}$ every 3 weeks). However, in our study the efficacy of treatment with pembrolizumab was comparable with those reported in previous studies (Table 2), and was consistent with the results of a study suggesting that low-dose pembrolizumab (100 mg every 3 weeks) could be effective for relapsed or refractory HL, with minimal toxicity [28]. In fact, when using this low dose of pembrolizumab we observed six responders (2 CR, 4 PR) out of eight patients with relapsed or refractory HL who failed treatment with brentuximab vedotin and autologous SCT (ORR, 75\%) (S1 Table). The outcome of our low-dose treatment was comparable to that of previous phase II studies of relapsed or refractory HL that reported an ORR of $65 \%$ (16\% CR) using $10 \mathrm{mg} / \mathrm{kg}$ of pembrolizumab every 2 weeks [29] and an ORR of $69 \%$ ( $22.4 \%$ CR) using $200 \mathrm{mg}$ of pembrolizumab every 3 weeks [30].

None of the patients in our study showed any grade 3 or 4 hematologic or nonhematologic toxicities, and their causes of death were related to disease progression. Although one patient developed a bowel perforation, that patient had previously experienced bowel perforation during salvage che- motherapy before initiation of pembrolizumab treatment. Therefore, the toxicity of pembrolizumab was manageable, which might be because we used a low dose of $100 \mathrm{mg}$ of pembrolizumab every 3 weeks, although a previous study using $10 \mathrm{mg} / \mathrm{kg}$ of pembrolizumab every 2 weeks reported five cases of grade 3 drug-related adverse events (16\%). Furthermore, five patients in our study had undergone unrelated or haploidentical allogeneic SCT before receiving pembrolizumab, but only one patient developed grade II acute GVHD (case 1 with NKTCL) (Fig. 1). This might be related to the lower dose of pembrolizumab and be consistent with the safety of PD1 blockade after allogeneic SCT [10].

In conclusion, pembrolizumab could be useful as a salvage treatment for relapsed or refractory EBV-positive NHL, especially NKTCL. However, its efficacy in EBV-negative NHL with low or absent PDL1 expression is still not clear although pembrolizumab could be a potential treatment option for relapsed or refractory NHL.

\section{Electronic Supplementary Material}

Supplementary materials are available at Cancer Research and Treatment website (https: // www.e-crt.org).

\section{Conflicts of Interest}

Conflict of interest relevant to this article was not reported.

\section{Acknowledgments}

This study was supported by a grant from the Basic Science Research Program through the National Research Foundation of Korea (NRF), which is funded by the Ministry of Education, Science, and Technology (NRF-2017R1A2B4005136). We also give thanks to all colleagues for participating in the research, and express our gratitude to the 20-20 project of the Samsung Medical Center.

\section{References}

1. Mak V, Hamm J, Chhanabhai M, Shenkier T, Klasa R, Sehn $\mathrm{LH}$, et al. Survival of patients with peripheral T-cell lymphoma after first relapse or progression: spectrum of disease and rare long-term survivors. J Clin Oncol. 2013;31:1970-6.

2. Crump M, Neelapu SS, Farooq U, Van Den Neste E, Kuruvilla $\mathrm{J}$, Westin J, et al. Outcomes in refractory diffuse large B-cell lymphoma: results from the international SCHOLAR-1 study. Blood. 2017;130:1800-8.

3. Weber J. Immune checkpoint proteins: a new therapeutic paradigm for cancer--preclinical background: CTLA-4 and PD-1 blockade. Semin Oncol. 2010;37:430-9.

4. Chen L, Flies DB. Molecular mechanisms of T cell co-stimulation and co-inhibition. Nat Rev Immunol. 2013;13:227-42.

5. Kwong YL, Chan TS, Tan D, Kim SJ, Poon LM, Mow B, et al. PD1 blockade with pembrolizumab is highly effective in relapsed or refractory NK/T-cell lymphoma failing l-asparaginase. Blood. 2017;129:2437-42.

6. Cheson BD, Fisher RI, Barrington SF, Cavalli F, Schwartz LH, Zucca E, et al. Recommendations for initial evaluation, staging, and response assessment of Hodgkin and non-Hodgkin 
lymphoma: the Lugano classification. J Clin Oncol. 2014;32: 3059-68.

7. Kim SJ, Choi JY, Hyun SH, Ki CS, Oh D, Ahn YC, et al. Risk stratification on the basis of Deauville score on PET-CT and the presence of Epstein-Barr virus DNA after completion of primary treatment for extranodal natural killer/T-cell lymphoma, nasal type: a multicentre, retrospective analysis. Lancet Haematol. 2015;2:e66-74.

8. Dako North America. PD-L1 IHC 22C3 pharmDx (package insert). Carpinteria, CA: Dako North America; 2015.

9. Lai J, Xu P, Jiang X, Zhou S, Liu A. Successful treatment with anti-programmed-death-1 antibody in a relapsed natural killer/T-cell lymphoma patient with multi-line resistance: a case report. BMC Cancer. 2017;17:507.

10. Chan TS, Khong PL, Kwong YL. Pembrolizumab for relapsed anaplastic large cell lymphoma after allogeneic haematopoietic stem cell transplantation: efficacy and safety. Ann Hematol. 2016;95:1913-5.

11. Zinzani PL, Ribrag V, Moskowitz CH, Michot JM, Kuruvilla J, Balakumaran A, et al. Safety and tolerability of pembrolizumab in patients with relapsed/refractory primary mediastinal large B-cell lymphoma. Blood. 2017;130:267-70.

12. Nastoupil LJ, Westin J, Fowler N, Fanale M, Samaniego F, Oki $\mathrm{Y}$, et al. High response rates with pembrolizumab in combination with rituximab in patients with relapsed follicular lymphoma: interim results of an on open-label, phase II study. Hematol Oncol. 2017;35(Suppl 2):120-1.

13. Chan TS, Khong PL, Kwong YL. Pembrolizumab and lenalidomide induced remission in refractory double-hit lymphoma. Ann Hematol. 2016;95:1917-8.

14. Menter T, Bodmer-Haecki A, Dirnhofer S, Tzankov A. Evaluation of the diagnostic and prognostic value of PDL1 expression in Hodgkin and B-cell lymphomas. Hum Pathol. 2016;54: 17-24.

15. Vranic S, Ghosh N, Kimbrough J, Bilalovic N, Bender R, Arguello D, et al. PD-L1 status in refractory lymphomas. PLoS One. 2016;11:e0166266.

16. Kiyasu J, Miyoshi H, Hirata A, Arakawa F, Ichikawa A, Niino $D$, et al. Expression of programmed cell death ligand 1 is associated with poor overall survival in patients with diffuse large B-cell lymphoma. Blood. 2015;126:2193-201.

17. Roemer MG, Advani RH, Ligon AH, Natkunam Y, Redd RA, Homer $\mathrm{H}$, et al. PD-L1 and PD-L2 genetic alterations define classical Hodgkin lymphoma and predict outcome. J Clin Oncol. 2016;34:2690-7.

18. Twa DD, Chan FC, Ben-Neriah S, Woolcock BW, Mottok A, Tan KL, et al. Genomic rearrangements involving programmed death ligands are recurrent in primary mediastinal large B-cell lymphoma. Blood. 2014;123:2062-5.

19. Chapuy B, Roemer MG, Stewart C, Tan Y, Abo RP, Zhang L, et al. Targetable genetic features of primary testicular and primary central nervous system lymphomas. Blood. 2016;127: 869-81.

20. Four M, Cacheux V, Tempier A, Platero D, Fabbro M, Marin $\mathrm{G}$, et al. PD1 and PDL1 expression in primary central nervous system diffuse large B-cell lymphoma are frequent and expression of PD1 predicts poor survival. Hematol Oncol. 2017;35: 487-96.

21. Jo JC, Kim M, Choi Y, Kim HJ, Kim JE, Chae SW, et al. Expression of programmed cell death 1 and programmed cell death ligand 1 in extranodal NK/T-cell lymphoma, nasal type. Ann Hematol. 2017;96:25-31.

22. Kim WY, Jung HY, Nam SJ, Kim TM, Heo DS, Kim CW, et al. Expression of programmed cell death ligand 1 (PD-L1) in advanced stage EBV-associated extranodal NK/T cell lymphoma is associated with better prognosis. Virchows Arch. 2016;469:581-90.

23. Ding W, LaPlant BR, Call TG, Parikh SA, Leis JF, He R, et al. Pembrolizumab in patients with CLL and Richter transformation or with relapsed CLL. Blood. 2017;129:3419-27.

24. Chen BJ, Chapuy B, Ouyang J, Sun HH, Roemer MG, Xu ML, et al. PD-L1 expression is characteristic of a subset of aggressive B-cell lymphomas and virus-associated malignancies. Clin Cancer Res. 2013;19:3462-73.

25. Roach C, Zhang N, Corigliano E, Jansson M, Toland G, Ponto $\mathrm{G}$, et al. Development of a companion diagnostic PD-L1 immunohistochemistry assay for pembrolizumab therapy in non-small-cell lung cancer. Appl Immunohistochem Mol Morphol. 2016;24:392-7.

26. Reck M, Rodriguez-Abreu D, Robinson AG, Hui R, Csoszi T, Fulop A, et al. Pembrolizumab versus chemotherapy for PD-L1-positive non-small-cell lung cancer. N Engl J Med. 2016; 375:1823-33.

27. Cheson BD, Ansell S, Schwartz L, Gordon LI, Advani R, Jacene $\mathrm{HA}$, et al. Refinement of the Lugano Classification lymphoma response criteria in the era of immunomodulatory therapy. Blood. 2016;128:2489-96.

28. Chan TS, Luk TH, Lau JS, Khong PL, Kwong YL. Low-dose pembrolizumab for relapsed / refractory Hodgkin lymphoma: high efficacy with minimal toxicity. Ann Hematol. 2017;96: 647-51.

29. Armand P, Shipp MA, Ribrag V, Michot JM, Zinzani PL, Kuruvilla J, et al. Programmed death-1 blockade with pembrolizumab in patients with classical Hodgkin lymphoma after brentuximab vedotin failure. J Clin Oncol. 2016;34:3733-9.

30. Chen R, Zinzani PL, Fanale MA, Armand P, Johnson NA, Brice $\mathrm{P}$, et al. Phase II study of the efficacy and safety of pembrolizumab for relapsed/refractory classic Hodgkin lymphoma. J Clin Oncol. 2017;35:2125-32. 